\title{
Dose Modification
}

National Cancer Institute

\section{Source}

National Cancer Institute. Dose Modification. NCI Thesaurus. Code C54346.

Change, alteration, or adjustment of a dose specified in a treatment plan or/and administered to a patient. 